\title{
The Role of the Atmosphere on the Photophysics of Ligand-Free Lead-Halide Perovskite Nanocrystals
}

\author{
María Morán-Pedroso, Andrea Rubino, Mauricio E. Calvo, Juan P. Espinós, \\ Juan F. Galisteo-López,* and Hernán Míguez**
}

Lead halide perovskite (LHP) nanocrystals (NCs) have gained attention over the past decade due to their outstanding optoelectronic properties, making them a suitable material for efficient photovoltaic and light emitting devices. Due to its soft nature, these nanostructures undergo strong structural changes upon irradiation, where these light-induced processes are strongly influenced by the environment. Since most processing routes for LHP NCs are based on colloidal approaches, the role of factors such as stabilizing ligands or solvents is usually hard to disentangle from the interaction of external radiation with the perovskite material. Employing a recently proposed synthetic approach, where ligand-free NCs can be grown within metal-oxide-based insulating nanoporous matrices, it has been feasible to perform a clean study of the effect of the surrounding atmosphere on the photophysical properties of perovskite NCs, avoiding the interference of protective capping layers or solvents. Simultaneous light-induced photo-activation and darkening processes are monitored and disentangled, and their relation with bulk and surface processes, respectively, demonstrated.

\section{Introduction}

The potential for lead-halide perovskites (LHP) as light emitters stems from their enhanced binding energy upon a reduction of its size, making its excited species of an excitonic nature and improving its radiative efficiency. ${ }^{[1]}$ This turns LHP nanocrystals (NCs) into a key element for applications in light emitting devices, ${ }^{[2]}$ whose potential is further enhanced by other aspects such as the possibility of tuning its electronic bandgap across the entire visible by appropriate combination of its halide component ${ }^{[3,4]}$ or a slow cooling of hot carriers in LHP nanostructures ${ }^{[5]}$

M. Morán-Pedroso, Dr. A. Rubino, Dr. M. E. Calvo, Prof. J. P. Espinós, Dr. J. F. Galisteo-López, Prof. H. Míguez

Instituto de Ciencia de Materiales de Sevilla

Consejo Superior de Investigaciones Científicas-Universidad de Sevilla

C/Américo Vespucio 49, Sevilla 41092, Spain

E-mail: juan.galisteo@csic.es; h.miguez@csic.es

The ORCID identification number(s) for the author(s) of this article can be found under https://doi.org/10.1002/adom.202100605.

(C) 2021 The Authors. Advanced Optical Materials published by Wiley$\mathrm{VCH} \mathrm{GmbH}$. This is an open access article under the terms of the Creative Commons Attribution-NonCommercial License, which permits use, distribution and reproduction in any medium, provided the original work is properly cited and is not used for commercial purposes.

DOI: 10.1002/adom.202100605 that could pave the way for their use in photovoltaic devices with efficiencies surpassing that of the Shockley-Queisser limit. ${ }^{[6]}$

But the photophysics of LHP are dictated by a series of complex processes taking place over several timescales from the generation, diffusion, and recombination of photo-excited carriers to slower processes involving the migration of ionic species. The latter processes are known to lead to photobrightening and photodarkening phenomena. Further, many of these processes are known to be strongly influenced by the surrounding atmosphere. ${ }^{[7]}$ While the ultimate mechanism behind these photo-induced changes is still under debate ${ }^{[8]}$ they play a key role in the emission properties as well as the long-term stability of LHP and thus on the performance of these materials in optoelectronic devices. These phenomena have been widely studied for LHP in the shape of single crystals and polycrystalline films comprising crystallites having sizes from hundreds of $\mathrm{nm}$ to a few microns, but their role in LHP nanostructures have been barely considered. ${ }^{[9,10]}$ Such study, involving the understanding of the interaction of light and matter in LHP for nanoscale systems, is usually an issue since most NCs are synthesized employing colloidalbased methods where NCs are dispersed in a liquid medium and surrounded by ligands that passivate defects, prevent their aggregation and control size and shape. Recently a novel approach based on the synthesis of LHP NCs within a dielectric porous matrix has been presented leading to the formation of ligand-free LHP NCs with outstanding emitting properties. ${ }^{[11-15]}$ Further, NCs fabricated following this approach have demonstrated enhanced stability as compared to their bulk counterparts. These LHP nanostructures represent an excellent platform to study the photophysics of ligand-free NCs as their size is reduced down to the quantum confinement limit. ${ }^{[16]}$

Here we present a study of the photophysics of ligand-free $\mathrm{MAPbBr}_{3} \mathrm{NCs}$ of different size grown within $\mathrm{SiO}_{2}$ nanoporous matrices and exposed to different levels of atmospheric oxygen. Photoactivation and photo-darkening processes are observed to take place simultaneously and the dominance of either is shown to strongly depend on the size of the crystal and the presence of neighboring crystals, pointing to the presence of surface-related degradation and bulk-related photo-activation. To further understand the role of the atmosphere on the two optical processes, 
structural information is retrieved from X-ray photoemission spectroscopy (XPS) measurements. Finally, the existence of two photo-induced processes involving different timescales and of different nature are unveiled. This study highlights the particularity of the photophysics of LHP NCs as compared to those of the bulk material, as well as the relevance of NCs grown within nanoporous matrices, which allows studying the optical properties of LHP nanostructures in the absence of any external elements such as ligands.

\section{Results and Discussion}

\subsection{Photophysical Characterization}

$\mathrm{MAPbBr}_{3} \mathrm{NCs}$ of two different sizes $(2.35$ and $2.57 \mathrm{~nm}$ diameter) were grown within $\mathrm{SiO}_{2}$ nanoporous matrices following a previously reported procedure. ${ }^{[17,18]}$ The size of the NC, which determines its optical properties, can be controlled via the concentration of the precursors in the solution spin coated on the $\mathrm{SiO}_{2}$ matrix (see Section S1, Supporting Information). The photoluminescence (PL) from the different NCs matched with the onset of absorption (see Figure S3, Supporting Information $)^{[17]}$ and a blueshift of the PL peak was evident as the amount of precursor on the synthesis solution was reduced (Figure 1a). Next the emission properties of the samples were monitored as the amount of oxygen in the surrounding atmosphere was controlled in a precise manner. For this, the samples were placed inside a chamber where the atmosphere could be brought down to ultra-high vacuum (UHV) conditions $\left(10^{-8}\right.$ Torr $)$ or filled with controlled amounts of oxygen. To assess the role of the latter, the emission was studied under UHV as well as under different oxygen pressures $(2,4,6,8$ and 10 Torr). Figure 1b,c shows selected PL spectra from a $2.57 \mathrm{~nm}$ NC sample at different irradiation times under the two extreme cases of UHV and 10 Torr, where the role played by oxygen becomes patent as changes in the PL intensity but also in the spectral position.

As the following step, the intensity and spectral position of the emission was monitored for different samples as they were irradiated either under UHV or when exposed to 2 Torr of $\mathrm{O}_{2}$. Figure 2 shows the evolution of these parameters for the different NC samples as well as a bulk sample. Here it becomes evident that under UHV conditions (gray curves) the samples initially emit poorly and undergo a small PL darkening upon irradiation, regardless of their morphology, something already observed for bulk LHP materials of different composition. ${ }^{[19-21]}$ Such changes are not accompanied by any spectral variation. But when small amounts of oxygen are introduced into the sample chamber (black curves) the optical response is modified, changes being strongly dependent on sample morphology. After a small initial fast variation in PL taking place within the first minute of irradiation (see below), the bulk sample undergoes a strong PL activation followed by a posterior bleach (Figure 2a), similar to previous reports. ${ }^{[21-23]}$ For the larger NCs $(2.57 \mathrm{~nm})$, after the initial abrupt change a slower rise in PL is observed in a similar time-scale (Figure $2 \mathrm{~b}$ ). Finally for the smallest NCs $(2.35 \mathrm{~nm})$ only a drop in PL is observed (Figure 2c). Contrarily to what is observed for the bulk sample,

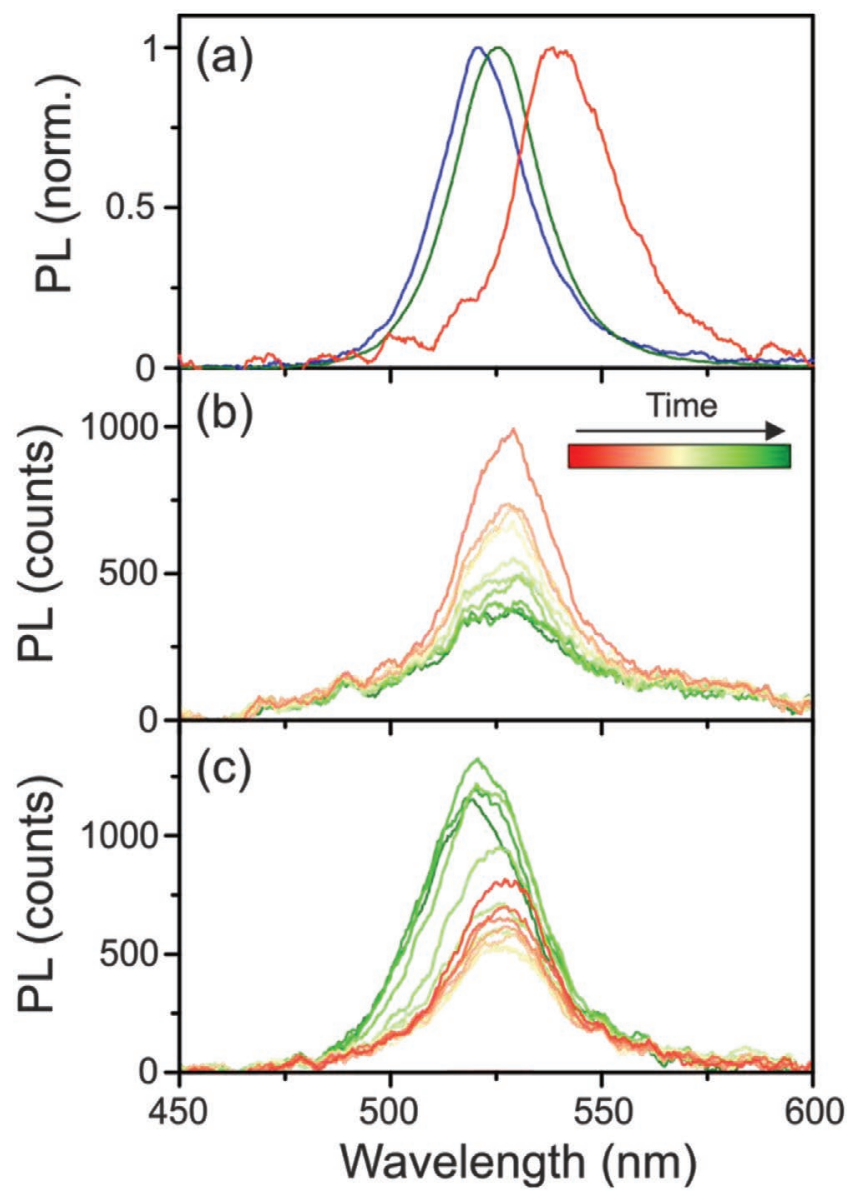

Figure 1. a) PL spectra of different samples under an oxygen rich atmosphere (10 Torr): $2.35 \mathrm{~nm} \mathrm{NCs} \mathrm{(blue),} 2.57 \mathrm{~nm} \mathrm{NCs} \mathrm{(green)} \mathrm{and} \mathrm{bulk} \mathrm{mate-}$ rial in the shape of a thin film (red curve). b,c) PL spectra at different times of irradiation for the $2.57 \mathrm{~nm} \mathrm{NC}$ sample placed under UHV and 10 Torr $\mathrm{O}_{2}$ atmospheres, respectively.

in the case of NCs changes in PL intensity are accompanied by a variation in the spectral position of the PL peak, which will be discussed later. These trends were observed for several sets of samples and over a large range of $\mathrm{O}_{2}$ pressures (see Figure $\mathrm{S} 5$, Supporting Information).

Regarding the duration of both effects, while photo-darkening could be associated with the permanent degradation of the material (see Section S2 and Figure S6, Supporting Information) the photo-activation is partially reversible over a time-span of several hours (see Figure 3). To asses this, the $2.57 \mathrm{~nm}$ NC sample was irradiated continuously in an $\mathrm{O}_{2}$ rich atmosphere (10 Torr) until its PL achieved the plateau just before darkening kicks in. Then it was kept in the dark and emission spectra were collected for $1 \mathrm{~s}$ (a short enough irradiation time in order not to introduce any appreciable activation effects) at different intervals: each $30 \mathrm{~s}$ initially and each $30 \mathrm{~min}$ in the last stage. In Figure 3 we can see how after $5 \mathrm{~h}$ in the dark the sample's PL has achieved a plateau retaining $75 \%$ of its maximum PL intensity. Further, we can also see how spectral changes (blueshift) accompanying the activation are also retained over the same time period. 

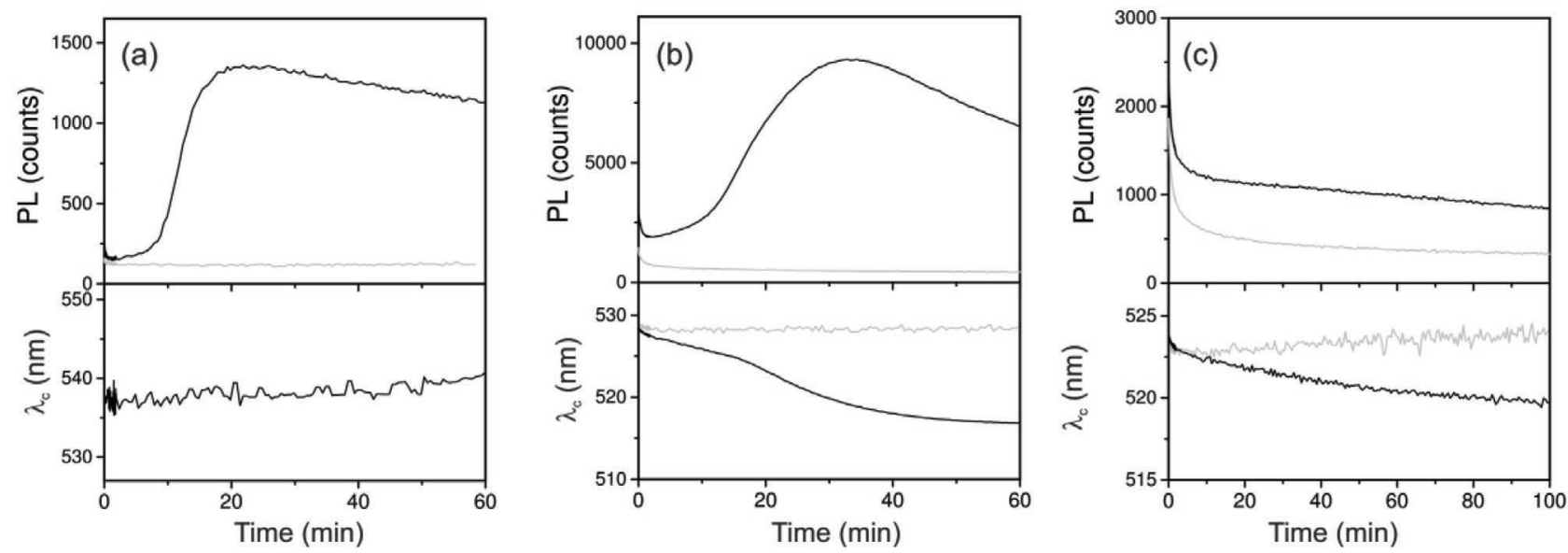

Figure 2. Time evolution of intensity and spectral position of the PL of samples of different morphology upon continuous irradiation under UHV (gray) and 2 Torr of oxygen (black curves): a) bulk, b) $2.57 \mathrm{~nm} \mathrm{NC}$, and c) $2.35 \mathrm{~nm} \mathrm{NC}$.

\subsection{Analysis of PL Activation and Darkening}

In order to rationalize the time evolution of the PL curves we assumed that the two photo-induced processes commonly reported for LHP - trap deactivation/annihilation and material degradation-are taking place simultaneously, hence competing with each other. ${ }^{[21]}$ The process of photo-activation is modeled with a logistic function (Equation (1)) which represents an initial PL $I_{0}$ which undergoes an activation with a growth rate $k_{\text {act }}$ as charge traps introducing non-radiative recombination paths for photogenerated carriers are deactivated (Figure 4a). For long enough times this population of traps is annihilated and the PL reaches a saturation value $I_{\text {sat }}=I_{0}+A$.

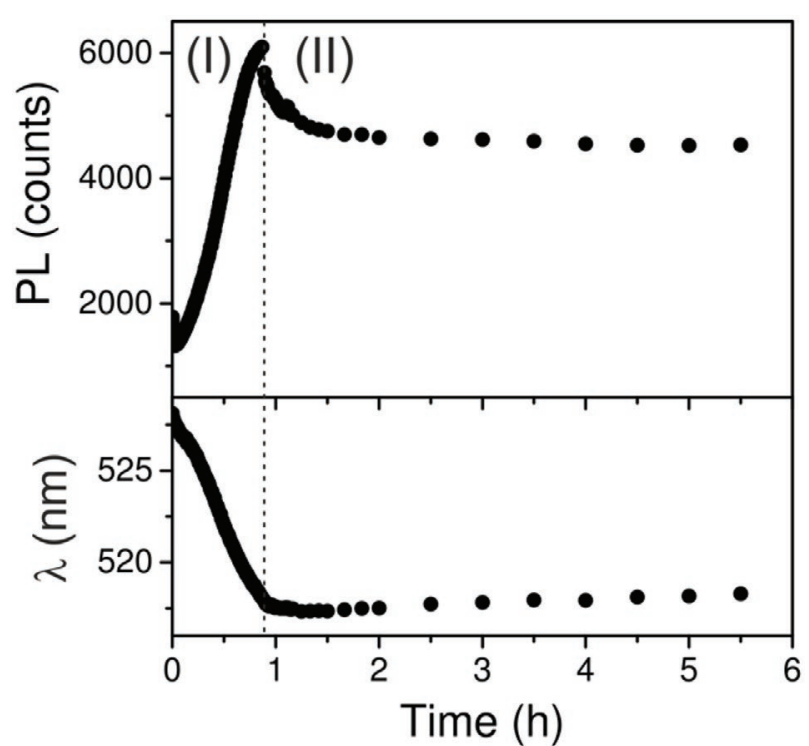

Figure 3. Time evolution of the maximum (top) and spectral position (bottom graph) of the $\mathrm{PL}$ from a $2.57 \mathrm{~nm} \mathrm{NC}$ sample in an $\mathrm{O}_{2}$-rich (10 Torr) atmosphere during continuous irradiation (I) and after being kept in the dark (II) for several hours.
$I(t)=I_{0}+\frac{A}{1+e^{-k_{a c t}\left(t-t_{c}\right)}}$

As it has been shown before, this process is accompanied by a photo-darkening which has been previously associated with material degradation induced by the presence of superoxide species formed under illumination in an oxygen atmosphere. ${ }^{[21]}$ This degradation is assumed to introduce an exponential drop in the PL intensity with a rate $k_{\text {decay }}$ (Figure $4 \mathrm{~b}$ ):

$I(t) \propto e^{-k_{\text {decay }} t}$

The experimental curves could then be well fitted (see Figure $4 \mathrm{c}$ ) to the product of the logistic and single exponentials from which values for $k_{\text {act }}$ and $k_{\text {decay }}$ are extracted. The evolution of the two rates is shown in Figure 4d,e as a function of $\mathrm{O}_{2}$ pressure. For the case of the bulk only pressures until 6 Torr are considered as for higher pressures the activation is too fast to be observed with the present time resolution. Here it can be clearly seen that the activation process (see Figure 4d) is clearly faster for the larger crystals present in the thin film. For the case of the smallest NCs $(2.35 \mathrm{~nm}$ sample) no data is shown as no activation is present (at least within the time range of the experiment). This clearly points to activation being a volume-related process that is involving the interior of the NC.

For the case of the PL darkening, in order to compare all NC sizes, the smallest $2.35 \mathrm{~nm}$ NCs were fitted to a double decay and an average $k_{\text {decay }}$ was extracted. Results are shown in Figure 4e. Here it is clearly seen that decay is faster for smaller samples pointing to photo-induced degradation being mainly a surface effect. It must be noted that for the smallest crystals, degradation is much faster than for the other NC size, not corresponding to their difference in size (see Table S1, Supporting Information). This can be related with the fact that increasing the precursor amount carries an increase in NC size but also of the NC load leading to aggregation and thus less surfaces are being exposed to the surrounding atmosphere. 

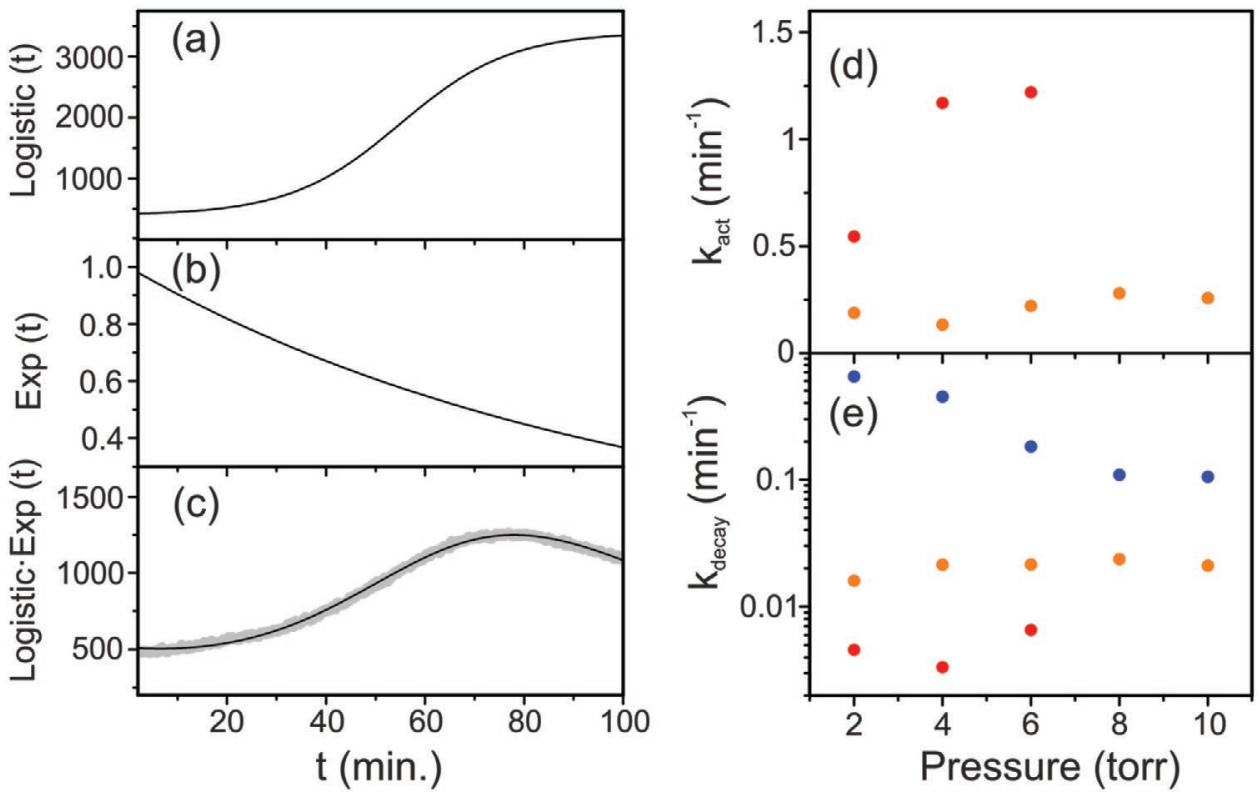

Figure 4. a) Time evolution of a logistic function, b) a single exponential decay, and c) its product when fitted to the experimental data (gray dots) for the case of the $2.57 \mathrm{~nm}$ NCs sample in a 2 Torr $\mathrm{O}_{2}$ atmosphere. Evolution of the d) activation $k_{\text {act }}$ and e) darkening $k_{\text {decay }}$ rate constants as a function of the $\mathrm{O}_{2}$ atmosphere for the different samples under consideration: $2.35 \mathrm{~nm} \mathrm{NCs}$ (blue), $2.57 \mathrm{~nm} \mathrm{NCs} \mathrm{(orange)} \mathrm{deposited} \mathrm{on} \mathrm{nanoporous} \mathrm{SiO}_{2}$ matrices as well as bulk material (red points).

\subsection{Analysis of the Chemical Structure: In Situ XPS Characterization}

In order to gain insight into the microscopic origin of these photo-induced processes we performed XPS studies at different stages of the irradiation. While for bulk $\mathrm{MAPbBr}_{3}$ XPS mainly provides information on changes taking place at the sample surface due to its limited escape depth (around $3 \lambda=7.2 \mathrm{~nm}$ ), ${ }^{[24]}$ in the present case the whole NC is probed due to its reduced size and thus changes taking place not only at the NC surface but also within its interior are expected to show in the XPS spectra. Further, in the present case no information on the presence of $\mathrm{O}_{2}$ related species at the $\mathrm{NC}$ surface can be obtained due to the XPS signal being dominated by the O1s signal from the $\mathrm{SiO}_{2}$ nanoporous matrix (see Figure S7, Supporting Information) contrary to the bulk case. ${ }^{[21]}$ The main photoemission signals associated with $\mathrm{Br}$ and $\mathrm{Pb}$ species (associated with $\mathrm{Br} 3 \mathrm{~d}$ and $\mathrm{Pb} 4 \mathrm{f}_{7 / 2}$ ) can be tracked (see Figure $5 \mathrm{a}, \mathrm{b}$ ) from which information on the evolution of the $\mathrm{Br} / \mathrm{Pb}$ atomic ratio can be extracted. This allows us to see that, opposite to previous experiments on bulk samples, ${ }^{[15]}$ the NC present a slight over-stoichiometric $\mathrm{Br}$ / $\mathrm{Pb}$ ratio (see Table S2 and discussion in Section S3, Supporting Information).

Next, XPS spectra were taken for two samples at different stages of the irradiation under an oxygen-rich atmosphere of 10 Torr. In both cases samples containing the highest precursor load (i.e. $2.57 \mathrm{~nm} \mathrm{NCs}$ ) were used to ensure a good XPS signal. The duration of each cycle was different in order to be in the time region where photo-induced PL activation or darkening dominates, respectively, which was monitored with the timeevolution of the sample PL (see Figure S8, Supporting Information). In both cases we can see a slight shift to lower energies of the $\mathrm{Br}$ and $\mathrm{Pb}$ signals (Figure $5 \mathrm{c}, \mathrm{d}$ show data for the degraded sample, for the activated one results are shown in Figure S9, Supporting Information) upon irradiation, which can be associated with a difference in charging effects at both the LHP and the metal oxide matrix. For the case of the PL activated sample, the $\mathrm{Br} / \mathrm{Pb}$ ratio changes within the variability of XPS measurements for a given sample (see Table S3, Supporting Information), which indicates that no significant changes occur. On the contrary, for the sample for which irradiation is carried out to the point of irreversible photo-darkening, the $\mathrm{Br} / \mathrm{Pb}$ ratio drops by a factor 1.32 (larger than the variability of this ratio for a given sample) below the stoichiometric value of 3 (from 3.96 to 2.74) pointing to a loss of $\mathrm{Br}$ during the degradation process similar to that reported previously for the bulk material. ${ }^{[21]}$

\subsection{Discussion}

As evidenced from the above data, photophysical properties of LHP NCs are dictated by simultaneous photo-induced PL reversible activation and irreversible darkening. The former is clearly a volume-related process becoming more evident for the bulk sample (see Figure 4d). Also, it is strongly influenced by the presence of $\mathrm{O}_{2}$, particularly for the bulk sample where $k_{\text {act }}$ increases with the $\mathrm{O}_{2}$ pressure and eventually saturates. Further, the large variations observed in $k_{\text {act }}$ for NCs of similar diameter but different concentration points to a process that involves intra but also inter-particle phenomena. This becomes clear when we consider the two NC samples under study, where increasing the precursor concentration by a sevenfold factor slightly rises the NC diameter by $10 \%$ but should increase much more the NC load and thus its connectivity.

The activation of the PL in a semiconducting material is associated with the removal of non-radiative recombination 

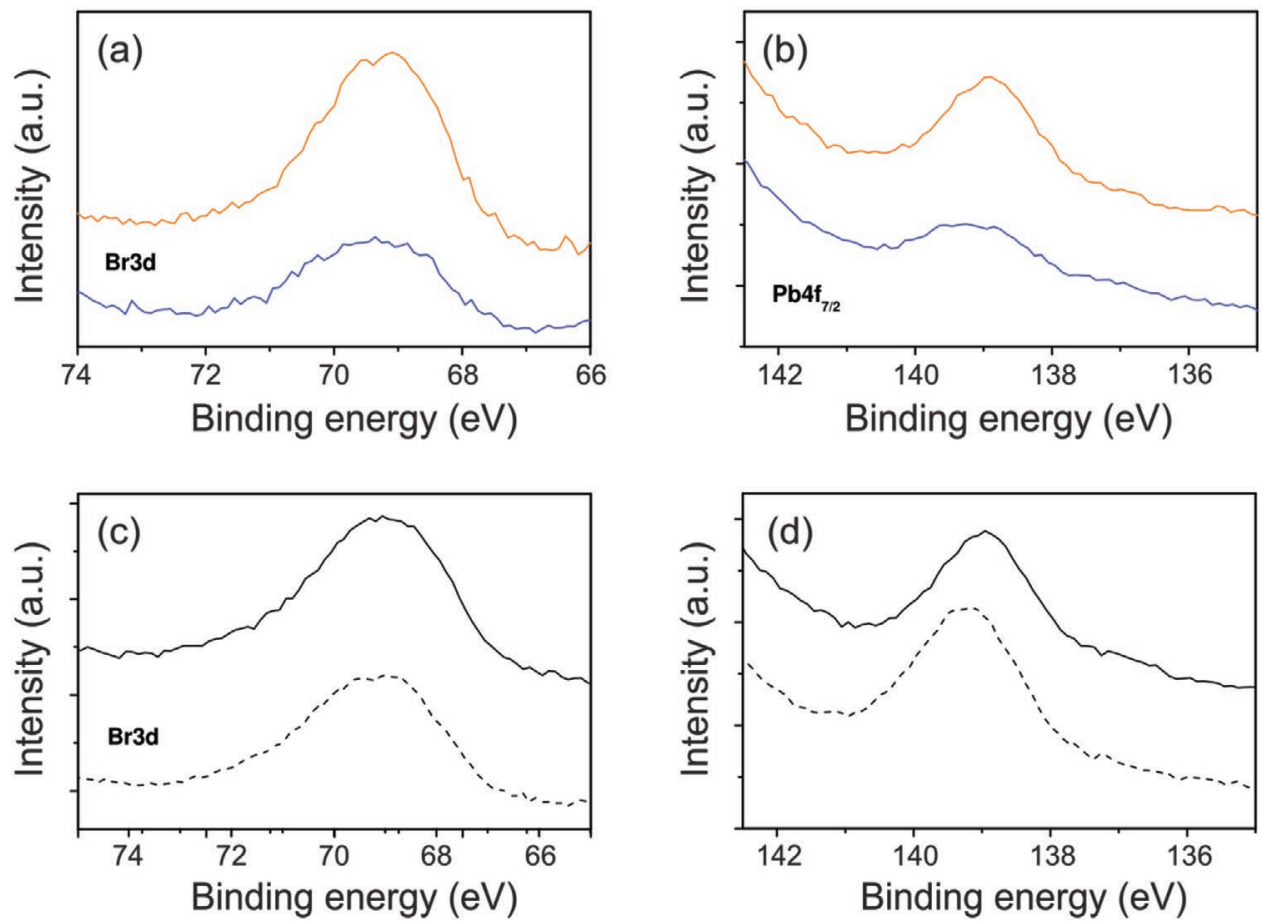

Figure 5. a) $\mathrm{Br} 3 \mathrm{~d}$ and b) $\mathrm{Pb} 4 \mathrm{f}_{7 / 2}$ photoelectron signals for $\mathrm{NC}$ samples having different diameters: $2.35 \mathrm{~nm}$ (blue) and $2.57 \mathrm{~nm}$ (orange curve). c) Br $3 \mathrm{~d}$ and d) $\mathrm{Pb} 4 \mathrm{f}_{7 / 2}$ photoelectron signals for the $2.57 \mathrm{~nm}$ sample as grown (black solid) and irradiated in 10 Torr of $\mathrm{O}_{2}$ (dashed curves) to the point of $\mathrm{PL}$ darkening treatment.

paths for photo-generated carriers. Such paths are caused by trap states located deep within the semiconductor bandgap which, for the case of bulk $\mathrm{MAPbX}_{3}(\mathrm{X}=\mathrm{I}, \mathrm{Br}$, and $\mathrm{Cl})$, have been associated mostly with halide-related interstitials. ${ }^{[25-27]}$ Several reports have also highlighted the role of surface halide traps in quenching the material emission (see below). Further, the modification of the defect structure of $\mathrm{MAPbX}_{3}$ under external irradiation has been thoroughly studied over the past years with an emphasis on the role played by the surrounding atmosphere. ${ }^{[19,20,21-23,28,29-35]}$ From this theoretical and experimental work, a complex light-mediated interaction of the LHP material with its environment emerges. While some works have reported emission quenching of $\mathrm{MAPbX}_{3}$ films in the presence of light and oxygen, ${ }^{[36,37]}$ the vast majority of experimental and theoretical work points to the role of oxygen combined with external illumination as the origin of photo-brightening in this material. Regarding the defect structure associated with deep energy traps, the main mechanisms proposed to explain the ubiquitous PL enhancement comprise the direct interaction of atmospheric oxygen with halide-related defects, either inducing a change in the oxidation state of bulk halide interstitials ${ }^{[27,31]}$ or passivating halide vacancies located at the sample surface. ${ }^{[29,32,34,38]}$ Other proposed mechanisms comprise the formation of secondary species such as $\mathrm{H}_{2} \mathrm{O}_{2}$ which oxidizes hole trapping atomic lead $\mathrm{Pb}^{0}{ }^{\left[{ }^{[33]}\right.}$ Finally, other routes have been suggested where Frenkel pairs comprising halide vacancies and interstitials are annihilated upon material irradiation. ${ }^{[21,28,35]}$ Such passivation of halide-related defects in the presence of oxygen has been further shown to be a means of preventing and even reversing the concomitant phase segregation effect in mixed LHP ${ }^{[39,40]}$ mediated by these types of defects.

While all of the above-mentioned scenarios may be taking place in the present case, the dominating PL activation mechanism is clearly one involving long-range inter-particle processes as evidenced from the lack of activation in NCs more prone to be isolated due to the low material load. Therefore the migration of halide species across different NCs leading to the annihilation of Frenkel pairs is the most plausible dominating mechanism. This coincides with the recent observation of halide ions migrating between different bulk LHP crystals placed in physical contact ${ }^{[4]}$ although in this latter case the driving force for the migration of the halide component was the entropy of mixing instead. Being strongly influenced by the presence of an $\mathrm{O}_{2}$-rich atmosphere, it is this molecular species the one likely to introduce a driving force for such migration, probably in the form of a negatively charged layer on the material surface as recently demonstrated for the bulk. ${ }^{[21]}$

PL darkening on the other hand is a surface dominated process as, under identical irradiation conditions, its rate increases for samples with a larger surface exposed to the environment (see Figure 3b). Its rate seems to be independent from the $\mathrm{O}_{2}$ pressure apart from the case of the small isolated NCs where a slight decrease in $k_{\text {act }}$ could be due to the presence of a PL enhancement too faint to show in the trend of Figure 2c. XPS data collected for a degraded NC sample (see Figure 5e,f) shows some similarities with the case of bulk samples. ${ }^{[21]}$ While appreciable sign of $\mathrm{Pb}^{0}$ is not seen, this can be due to the overall low signal retrieved due to the small amount of material within the nanoporous matrix. On the other hand, the $\mathrm{Br} / \mathrm{Pb}$ ratio drops by 
$30 \%$, in accordance to the bulk case where bromine was assumed to be lost as $\mathrm{Br}_{2}$. While negatively charged oxygen species are certainly playing a role in PL darkening, inducing the degradation of the LHP lattice, ${ }^{[21,42-44]}$ other processes involving the formation of molecular halide species that stabilize at the sample surface and induce material degradation may be present. ${ }^{[35]}$

For all-inorganic LHP with general formula $\mathrm{CsPb}_{3}$, many reports have shown a similar phenomenology regarding an improved emission in the presence of oxygen and light irradiation ${ }^{[9,45-47]}$ pointing to the universality of these processes. Nevertheless, for the case of NCs with this composition, illumination in an oxygen-rich atmosphere leads to a temporary quenching of their PL. ${ }^{[10,45,46]}$ The mechanism behind this has been associated with oxygen molecules adsorbed to the NC surface acting as electron scavengers, ${ }^{[10]}$ which could be related with their particular surface termination being different from that of larger structures. The fact that such quenching is fully reversible once the surrounding atmosphere is removed points to a different origin from the permanent degradation reported in the present work.

Finally, a difference in the distribution of defects between the two NC populations could contribute to the observed photophysical changes. Assuming that the nature and density of defects is similar in both cases, as synthesis conditions and precursor ratios are maintained, the main difference would be in the ratio between surface/bulk defects being related to degradation/activation, respectively. The surface-to-volume ratio increases by $10 \%$ when going from the large to the small NCs. Such difference is not expected to account for the large variations observed in PL activation/decay (see Figure 4). Nevertheless the possibility of smaller NCs, grown from much lower precursor deposition, presenting a larger defect density at the surface cannot be ruled out as an additional contribution to their fastest degradation upon light irradiation

One key ingredient in these photo-induced phenomena is the abovementioned presence of a blueshift pointing to a structural modification of the LHP lattice. This spectral shift, similar to that reported for photo-activated microscopic regions in single $\mathrm{MAPbBr}_{3}$ crystals, ${ }^{[48]}$ is only present in the NC systems (see Figure 2). As activation and darkening take place simultaneously, it is difficult to associate this spectral change with either process. Nevertheless, the fact that the blueshift follows the partial recovery behavior of the activation (see Figure 3) makes us think that it is likely related with the passivation of deep traps. Further, the absence of spectral changes when samples are irradiated in UHV and undergo a small PL drop further supports this point. Other light irradiation-related processes leading to structural modifications such as the recently reported photostriction ${ }^{[49,50]}$ can be ruled out as it involves spectral changes taking place in the opposite direction. Also a temperature induced spectral change, which would also lead to a blueshift, ${ }^{[51]}$ is not expected to be behind our observations as it would not remain for hours after irradiation is removed (see Figure 3). Photo-corrosion, which would indeed lead to a reduction in NC size, is not likely to be responsible for these spectral changes as it would lead to a reduction in emissive material which goes against the drastic PL enhancement observed. Hence the observed blueshift is most plausibly related to a reduction of lattice parameter accompanying the annihilation of crystalline defects. Such spectral change is only observed for the case of single crystals, either NCs or micron sized structures as reported in the past, ${ }^{[48]}$ while for the case of the polycrystalline films the presence of grain boundaries may compensate the strain release during defect passivation.

Finally, we would like to point out the existence of a faster photo-induced process taking place within the first minute of irradiation of the samples (see Figure 2 and Figure S2, Supporting Information) and only visible for high pump intensities (Figure S10, Supporting Information). Zooming into the PL changes in this time region we can see that although the variation in PL intensity can be similar to that observed for longer times, no spectral change takes place (Figure S11, Supporting Information) pointing to a different origin. While at the moment we cannot propose an explanation for these changes, its relation with thermal processes or charge trapping can be ruled out as the PL can fully recover back to its original value (Figure S12, Supporting Information) over times shorter than the posterior activation but much larger than those expected for material cooling or charge de-trapping.

\section{Conclusions}

In summary the results here presented lead to a complex picture following the irradiation of LHP NCs in the presence of an $\mathrm{O}_{2}$-rich atmosphere where the dominating processes are a bulk defect annihilation and surface material degradation taking place simultaneously. The fact that the former is only observed for samples where NC aggregation is expected indicates that the process leading to trap annihilation, that is, the migration of halide-related species, takes place over distances exceeding the NC size. Further, the presence of a marked blueshift accompanying changes in PL intensity point to strong structural changes that remain over large periods of several hours. As recently proposed ${ }^{[8]}$ this paves the way to the use of light treatments under an adequate and controlled atmosphere to improve the performance of LHP by selecting the appropriate irradiation duration to maximize the activation of PL. In this sense, further work will be needed to understand the structural changes introduced in order to retain its duration which has been seen to vary from tens of seconds in the case of large single crystals ${ }^{[48]}$ to several hours for the case of NCs and polycrystalline films. ${ }^{[29]}$

\section{Supporting Information}

Supporting Information is available from the Wiley Online Library or from the author.

\section{Acknowledgements}

Financial support of the Spanish Ministry of Science and Innovation under grant MAT2017-88584-R (AEI/FEDER,-UE) is gratefully acknowledged.

\section{Conflict of Interest}

The authors declare no conflict of interest. 


\section{Data Availability Statement}

Research data are not shared.

\section{Keywords}

lead-halide perovskites, nanocrystals, photophysical properties

Received: March 24, 2021

Revised: May 18, 2021

Published online:

[1] N. Droseros, G. Longo, J. C. Brauer, M. Sessolo, H. J. Bolink, N. Banerji, ACS Energy Lett. 2018, 3, 1458.

[2] H. Cho, S. H. Jeong, M. H. Park, Y. H. Kim, C. Wolf, C. L. Lee, J. Y. Heo, Science 2015, 350, 1222.

[3] J. H. Noh, S. H. Im, J. H. Heo, T. N. Mandal, S. I. Seok, Nano Lett. 2013, 13, 1764.

[4] L. Protesescu, S. Yakunin, M. I. Bodnarchuk, F. Krieg, R. Caputo, C. H. Hendon, R. X. Yang, A. Walsh, M. V. Kovalenko, Nano Lett. 2015, 15, 3692.

[5] M. Li, J. Fu, Q. Xu, T. C. Sum, Adv. Mater. 2019, 31, 1802486.

[6] R. T. Ross, A. J. Nozik, J. Appl. Phys. 1982, 53, 3813.

[7] J. F. Galisteo-López, M. E. Calvo, H. Míguez, Adv. Opt. Mater. 2021, 9, 2100133.

[8] Z. Andaji-Garmaroudi, M. Anaya, A. J. Pearson, S. D. Stranks, Adv. Energy Mater. 2020, 10, 1903109

[9] D. Lu, Y. Zhang, M. L. Lai, A. Lee, C. L. Xie, J. Lin, T. Lei, Z. N. Lin, C. S. Kley, J. M. Huang, E. Rabani, P. Yang, Nano Lett. 2018, 18, 6967.

[10] M. Lorenzon, L. Sortino, Q. Akkerman, S. Accornero, J. Pedrini, M. Prato, V. Pinchetti, F. Meinardi, L. Manna, S. Brovelli, Nano Lett. 2017, 17, 3844.

[11] D. N. Dirin, L. Protesescu, D. Trummer, I. V. Kochetygov, S. Yakunin, F. Krumeich, N. P. Stadie, M. V. Kovalenko, Nano Lett. 2016, 16, 5866.

[12] V. Malgras, S. Tominaka, J. W. Ryan, J. Henzie, T. Takei, K. Ohara, Y. Yamauchi, J. Am. Chem. Soc. 2016, 138, 13874.

[13] M. Anaya, A. Rubino, T. C. Rojas, J. F. Galisteo-López, M. E. Calvo, H. Míguez, Adv. Opt. Mater. 2017, 5, 1601087.

[14] S. Demchyshyn, J. M. Roemer, H. Groiß, H. Heilbrunner, C. Ulbricht, D. Apaydin, A. Böhm, U. Rütt, F. Bertram, G. Hesser, Sci. Adv. 2017, 3, e1700738.

[15] A. Rubino, A. Francisco-López, A. J. Barker, A. Petrozza, M. E. Calvo, A. R. Goñi, H. Míguez, J. Phys. Chem. Lett. 2021, 12, 569.

[16] A. Rubino, L. Caliò, A. García-Bennett, M. E. Calvo, H. Míguez, Adv. Opt. Mater. 2020, 8, 1901868.

[17] A. Rubino, M. Anaya, J. F. Galisteo-López, T. C. Rojas, M. E. Calvo, H. Míguez, ACS Appl. Mater. Interfaces 2018, 10, 38334.

[18] D. O. Tiede, A. Rubino, M. E. Calvo, J. F. Galisteo-López, H. Míguez, J. Phys. Chem. C 2020, 124, 8041.

[19] H.-H. Fan, S. Adjokatse, H. Wei, J. Yang, G. R. Blake, J. Huang, J. Even, M. A. Loi, Sci. Adv. 2016, 2, e1600901.

[20] S. G. Motti, M. Gandini, A. J. Barker, J. M. Ball, A. R. Srimath Kandada, A. Petrozza, ACS Energy Lett. 2016, 1, 726.

[21] M. Anaya, J. F. Galisteo-Lop'ez, M. E. Calvo, J. P. Espinos, H. Míguez, J. Phys. Chem. Lett. 2018, 9, 3891.

[22] J. F. Galisteo-López, M. Anaya, M. E. Calvo, H. Míguez, J. Phys. Chem. Lett. 2015, 6, 2200.
[23] Y. Tian, M. Peter, E. Unger, M. Abdellah, K. Zheng, T. Pullerits, A. Yartsev, V. Sundström, I. G. Scheblykin, Phys. Chem. Chem. Phys. 2015, 17, 24978.

[24] S. Tanuma, C. J. Powell, D. R. Penn, Surf. Interface Anal. 1993, 20, 77.

[25] M. H. Du, J. Phys. Chem. Lett. 2015, 6, 1461

[26] S. G. Motti, D. Meggiolaro, S. Martani, R. Sorrentino, A. J. Barker, F. De Angelis, Adv. Mater. 2019, 31, 1901183

[27] J. He, W.-H. Fang, R. Long, Chem. Sci. 2019, 10, 10079.

[28] E. Mosconi, D. Meggiolaro, H. J. Snaith, S. D. Stranks, F. De Angelis, Energy Environ. Sci. 2016, 9, 3180

[29] R. Brenes, D. Guo, A. Osherov, N. Noel, C. Eames, E. M. Hutter, S. K. Pathak, F. Niroui, R. H. Friend, M. S. Islam, H. J. Snaith, V. Bulović, T. J. Savenije, S. D. Stranks, Joule 2017, 1, 155.

[30] X. Feng, H. Su, Y. Wu, H. Wu, J. Xie, X. Liu, J. Fan, J. Dai, Z. He, J. Mater. Chem. A 2017, 5, 12048.

[31] D. Meggiolaro, E. Mosconi, F. De Angelis, ACS Energy Lett. 2017, 2, 2794.

[32] R. Brenes, C. Eames, V. Bulovic, M. S. Islam, S. D. Stranks, Adv. Mater. 2018, 30, 1706208

[33] J. S. W. Godding, A. J. Ramadan, Y.-H. Lin, K. Schutt, H. J. Snaith, B. Wenger, Joule 2019, 3, 2716.

[34] J. He, W. H. Fang, R. Long, O. V. Prezhdo, J. Am. Chem. Soc. 2019, $141,5798$.

[35] S. G. Motti, D. Meggiolaro, A. J. Barker, E. Mosconi, C. A. R. Perini, J. M. Ball, M. Gandini, M. Kim, F. De Angelis, A. Petrozza, Nat. Photonics 2019, 13, 532.

[36] W. Kong, A. Rahimi-Iman, G. Bi, X. Dai, H. Wu, J. Phys. Chem. C 2016, 120, 7606

[37] K. Mantulnikovs, A. Glushkova, M. Kollár, L. Forró, E. Horváth, A. Sienkiewicz, ACS Appl. Electron. Mater. 2019, 1, 2007.

[38] W. Li, Y.-Y. Sun, L. Li, Z. Zhou, J. Tang, O. V. Prezhdo, J. Am. Chem. Soc. 2018, 140, 15753

[39] A. J. Knight, A. D. Wright, J. B. Patel, D. P. McMeekin, H. J. Snaith, M. B. Johnston, L. M. Herz, ACS Energy Lett. 2019, 4, 75.

[40] W. Fan, Y. Shi, T. Shi, S. Chu, W. Chen, K. O. Ighodalo, J. Zhao, K. Li, Z. Xiao, ACS Energy Lett. 2019, 4, 2052.

[41] T. Elmelund, R. A. Scheidt, B. Seger, P. V. Kamat, ACS Energy Lett. 2019, 4, 1961

[42] N. Aristidou, I. Sanchez-Molina, T. Chotchuangchutchaval, M. Brown, L. Martinez, T. Rath, S. A. Haque, Angew. Chem., Int. Ed. 2015, 54, 8208 .

[43] N. Aristidou, C. Eames, I. Sanchez-Molina, X. Bu, J. Kosco, M. S. Islam, S. A. Haque, Nat. Commun. 2017, 8, 15218.

[44] A. Senocrate, T. Acartürk, G. Y. Kim, R. Merkle, U. Starke, M. Grätzel, J. Maier, J. Mater. Chem. A 2018, 6, 10847.

[45] Y. Wang, Y. Ren, S. Zhang, J. Wu, J. Song, X. Li, J. Xu, C. H. Sow, H. Zeng, H. Sun, Commun. Phys. 2018, 1, 96.

[46] C. Rodà, A. L. Abdelhady, J. Shamsi, M. Lorenzon, V. Pinchetti, M. Gandini, F. Meinardi, L. Manna, S. Brovelli, Nanoscale 2019, 11, 7613.

[47] L. Qiao, R. Long, J. Phys. Chem. Lett. 2019, 10, 5499.

[48] J. F. Galisteo-López, M. E. Calvo, H. Míguez, ACS Appl. Energy Mater. 2019, 2, 6967.

[49] Y. Zhou, L. You, S. Wang, Z. Ku, H. Fan, D. Schmidt, A. Rusydi, L. Chang, L. Wang, P. Ren, L. Chen, G. Yuan, L. Chen, J. Wang, Nat. Commun. 2016, 7, 11193.

[50] H. Tsai, R. Asadpour, J.-C. Blancon, C. C. Stoumpos, O. Durand, J. W. Strzalka, B. Chen, R. Verduzco, P. M. Ajayan, S. Tretiak, J. Even, M. A. Alam, M. G. Kanatzidis, W. Nie, A. D. Mohite, Science 2018, 360,67 .

[51] R. L. Milot, G. E. Eperon, H. J. Snaith, M. B. Johnston, L. M. Herz, Adv. Funct. Mater. 2015, 25, 6218. 